STRUCTURE REPORTS

ISSN $1600-5368$

Received 27 September 2014

Accepted 1 October 2014

Edited by P. C. Healy, Griffith University, Australia

Keywords: crystal structure; 2-(4-chlorophenyl)2-oxoethyl 3-bromobenzoate; synthesis; $\pi-\pi$ interactions; inversion dimers

CCDC reference: 864789

Supporting information: this article has supporting information at journals.iucr.org/e

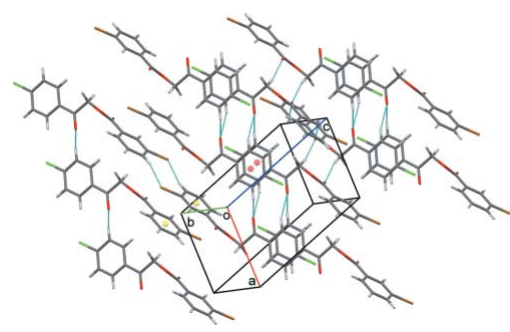

OPEN $\odot$ ACCESS

\section{Crystal structure of 2-(4-chlorophenyl)-2-oxoethyl 3-bromobenzoate}

\author{
Imtiaz Khan, ${ }^{\text {a,b }}$ Aliya Ibrar, ${ }^{\text {a }}$ Shahid Hameed, ${ }^{\mathrm{a}}$ Jonathan M. White ${ }^{\mathrm{c}}$ and Jim \\ Simpson $^{\mathrm{d} *}$
}

a Department of Chemistry, Quaid-i-Azam University, Islamabad 45320, Pakistan, ${ }^{\mathbf{b}}$ School of Chemistry, University of Nottingham, University Park, Nottingham NG7 2RD, England, 'School of Chemistry and Bio-21 Institute, University of Melbourne, Parkville, Victoria 3052, Australia, and dDepartment of Chemistry, University of Otago, PO Box 56, Dunedin, New Zealand. *Correspondence e-mail: jsimpson@alkali.otago.ac.nz

2-(4-Chlorophenyl)-2-oxoethyl 3-bromobenzoate, $\mathrm{C}_{15} \mathrm{H}_{10} \mathrm{BrClO}_{3}$, was synthesized in a single-step reaction by condensation of 3-bromobenzoic acid with 2-bromo-1-(4-chlorophenyl)ethanone in dimethylformamide in the presence of triethylamine as a catalyst. The structure consists of an aryl ketone moiety linked to an aryl ester unit by a methylene group. Both units are reasonably planar (r.m.s. deviations of 0.119 and $0.010 \AA$ for the aryl ketone and aryl ester units, respectively) and are almost orthogonal, with an angle of $88.60(3)^{\circ}$ between them. In the crystal, molecules form five separate sets of inversion dimers. Three of these are generated by two $\mathrm{C}-\mathrm{H} \cdots \mathrm{O}$ interactions and a $\mathrm{C}-$ $\mathrm{H} \cdot \mathrm{Br}$ contact, and form chains along $c$ and along the $a b$ cell diagonal. In addition, two inversion-related $\pi-\pi$ stacking interactions between like aryl rings again form chains of molecules but in this instance along the $b c$ diagonal. These contacts generate infinite layers of molecules parallel to (011) and stack the molecules along the $a$-axis direction.

\section{Chemical context}

Keto esters, an important class of versatile intermediates, have been reported to show antitumor activity against Ehrlich cells and HeLa cells (Kinoshita \& Umezawa, 1960). They also regulate the flowering times of some plants (Kai et al., 2007). Recent studies have revealed that they also exhibit inhibitory activity against two isozymes of $11 \beta$-hydroxysteroid dehydrogenases (11 $\beta$-HSD1 and $11 \beta$-HSD2), which catalyse the interconversion of active cortisol and inactive cortisone (Zhang et al., 2009). Dicarbonyl compounds and their derivatives are also among the most versatile and frequently employed synthons in organic synthesis, especially in heterocyclic chemistry (Stanovnik \& Svete, 2004; Sheibani et al., 2006a,b, 2007; Pal et al., 2008). In this work, we report the synthesis of 2-(4-chlorophenyl)-2-oxoethyl 3-bromobenzoate, (1), which may be used as an effective synthon in organic chemistry.<smiles>O=C(COC(=O)c1cccc(Br)c1)c1ccc(Cl)cc1</smiles>

\section{Structural commentary}

The structure of (1) consists of an aryl ketone moiety linked to an aryl ester unit by the C8 methylene group and both 


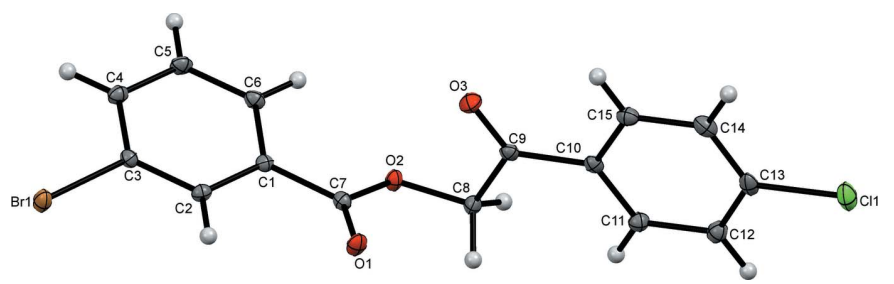

Figure 1

Fig, 1. The structure of (1) with displacement ellipsoids drawn at the $50 \%$ probability level.

groupings are reasonably planar. There is an r.m.s. deviation of $0.119 \AA$ from the best-fit plane through atoms Br1, C1-C8, O1, O2 [maximum deviation 0.2477 (11) $\AA$ for O1] while the plane of the carboxylate unit subtends an angle of $15.5(2)^{\circ}$ to that of the bromobenzene ring. In addition, the plane of the aryl ketone unit C8-C15, O3, Cl1 has an r.m.s. deviation of $0.010 \AA$ [maximum deviation 0.0171 (15) $\AA$ for C15]. The aryl ketone and aryl ester planes are almost orthogonal with an angle of $88.61(3)^{\circ}$ between them. Bond lengths and angles in the molecule are normal and are generally similar to those found

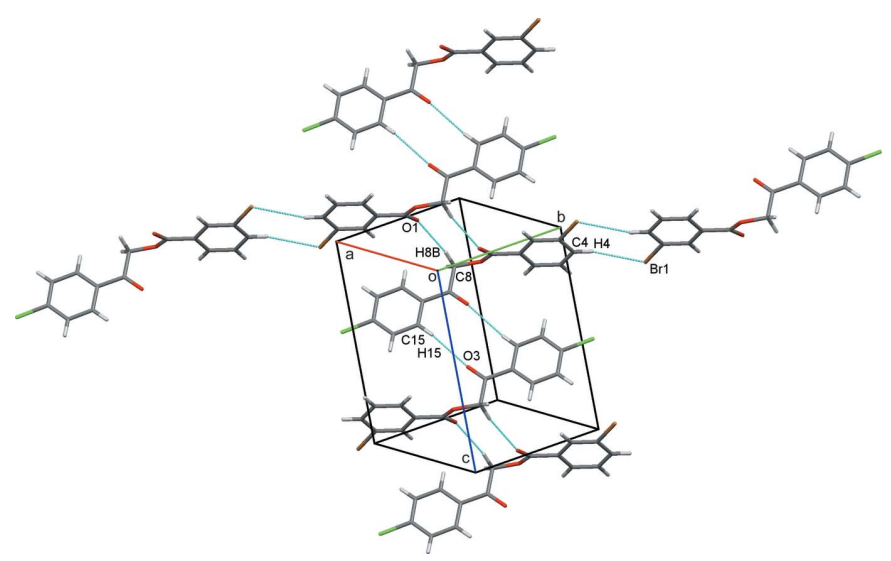

Figure 2

Chains of linked inversion dimers generated by $\mathrm{C}-\mathrm{H} \cdots \mathrm{O}$ and $\mathrm{C}-$ $\mathrm{H} \cdots \mathrm{Br}$ hydrogen bonds, drawn as dashed lines.

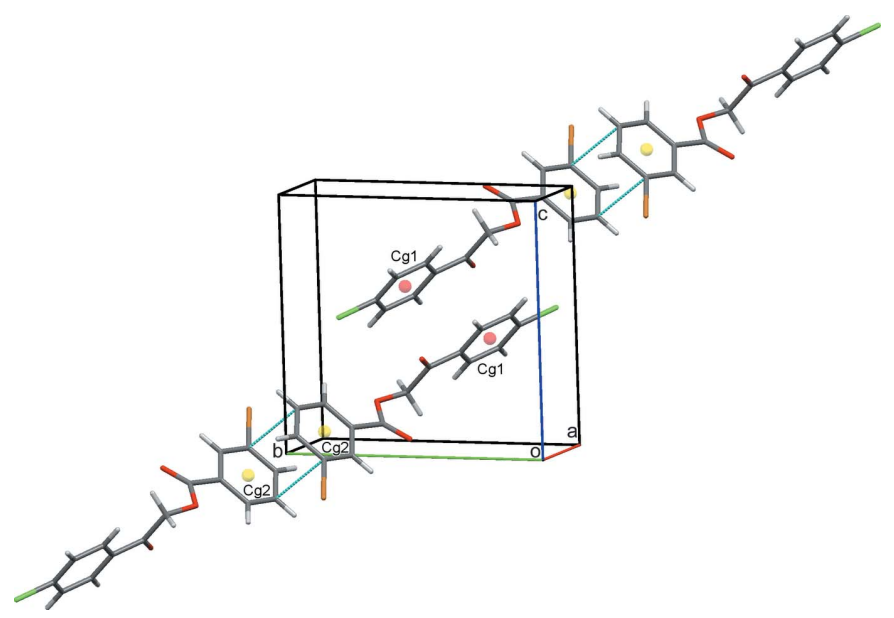

Figure 3

A chain of inversion dimers generated by $\pi-\pi$ contacts, dotted green lines, between 3-bromophenyl and 4-chlorophenyl rings. Ring centroids are displayed as coloured spheres.
Table 1

Hydrogen-bond geometry $\left(\AA{ }^{\circ}\right)$.

\begin{tabular}{|c|c|c|c|c|}
\hline$D-\mathrm{H} \cdots A$ & $D-\mathrm{H}$ & $\mathrm{H} \cdots A$ & $D \cdots A$ & $D-\mathrm{H} \cdots A$ \\
\hline $\mathrm{C} 4-\mathrm{H} 4 \cdots \mathrm{Br} 1^{\mathrm{i}}$ & 0.95 & 2.97 & $3.8762(18)$ & 160 \\
\hline $\mathrm{C} 8-\mathrm{H} 8 B \cdots \mathrm{O} 1^{\mathrm{ii}}$ & 0.99 & 2.42 & $3.396(2)$ & 168 \\
\hline $\mathrm{C} 15-\mathrm{H} 15 \cdots \mathrm{O}^{\mathrm{iii}}$ & 0.95 & 2.60 & $3.418(2)$ & 144 \\
\hline
\end{tabular}

in closely related molecules (see for example Fun et al., 2011a; Chidan Kumar et al., 2014c).

\section{Supramolecular features}

In the crystal structure, each molecule forms five separate inversion dimers. $\mathrm{C} 8-\mathrm{H} 8 B \cdots \mathrm{O} 1$ and $\mathrm{C} 15-\mathrm{H} 15 \mathrm{O} 3$ hydrogen bonds each generate $R_{2}^{2}(10)$ rings, forming zigzag chains along c. Additional $\mathrm{C} 4-\mathrm{H} 4 \cdots \mathrm{Br} 1$ contacts also form inversion dimers with $R_{2}^{2}(8)$ rings and these combine with the $\mathrm{C} 8-$ $\mathrm{H} 8 \mathrm{~B} \cdots \mathrm{O} 1$ contacts to link alternating pairs of dimers into infinite chains approximately along the $a b$ cell diagonal, Table 1, Fig. 2. Interestingly, infinite chains of alternating inversion dimers also result from a pair of $\pi-\pi$ stacking interactions between adjacent 3-bromophenyl rings, $C g 1 \cdots C g 1^{\text {iv }}=3.6987(10) \AA$, and neighbouring 4-chlorophenyl rings $C g 2 \cdots C g 2^{\mathrm{v}}=3.8585(11) \AA$, in this case along the $b c$ diagonal, Fig. 3 [ $C g 1$ and $C g 2$ are the centroids of the $\mathrm{C} 1-$ C6 and $\mathrm{C} 10-\mathrm{C} 15$ rings, respectively; symmetry codes (iv) $-x$, $2-y,-z$; (v) $2-x, 1-y, 1-z]$. These contacts combine to generate extended layers of molecules parallel to (011), Fig. 4, and to stack molecules along the $a$-axis direction, Fig. 5 .

\section{Database survey}

A search of the Cambridge Crystallographic Database (Groom \& Allen, 2014) reveals only eight structures with the 2-oxo-2-phenylethyl benzoate skeleton. These include the archetypal 2-oxo-2-phenylethyl benzoate (Fun et al., 2011a), three additional 2-(4-chlorophenyl)-2-oxoethyl derivatives (Fun et al., 2011b; Chidan Kumar et al., 2014a,b) and the

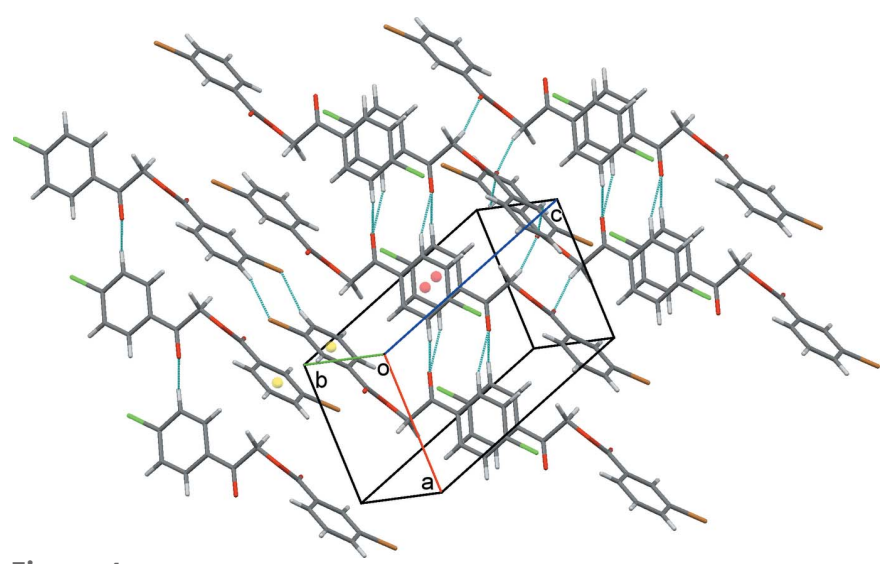

Figure 4

Overall packing of (1) viewed at right angles to (011). 


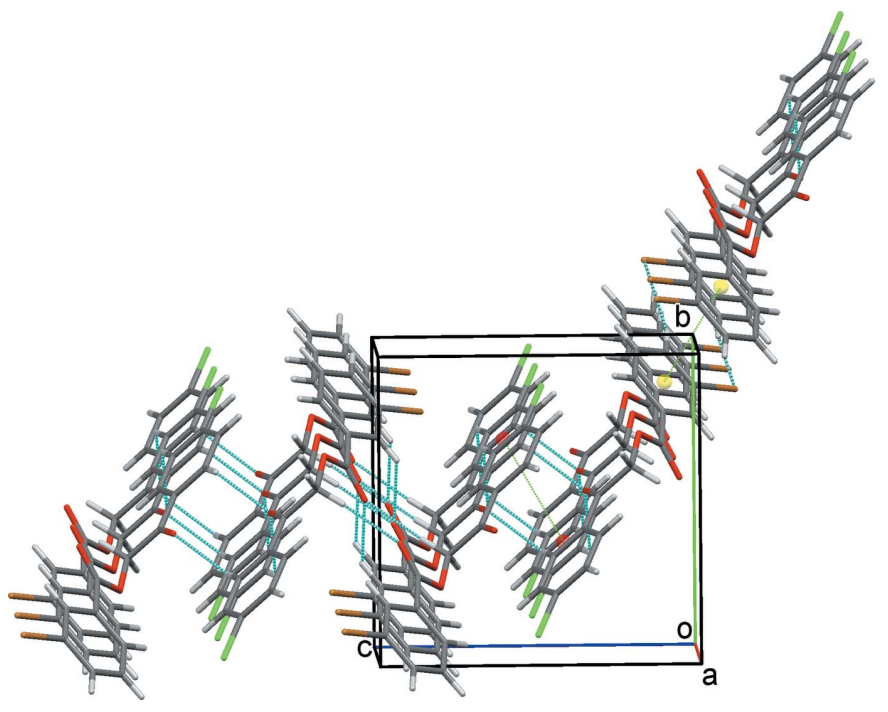

Figure 5

Overall packing of (1) viewed along the $a$-axis direction.

corresponding compound 2-(4-bromophenyl)-2-oxoethyl 3 -chlorobenzoate with the chloro- and bromo-substituents reversed (Chidan Kumar et al., 2014c). Interestingly, inversiondimer formation is a feature of the packing in several of these structures.

\section{Synthesis and crystallization}

The preparation followed a procedure developed for the preparation of a related compound (Khan et al., 2012). Triethylamine (4-5 drops) was added at room temperature to a stirred solution of 3-bromobenzoic acid $(1.0 \mathrm{mmol})$ in $N, N$-dimethylformamide (DMF), followed by a solution of 2-bromo-1-(4-chlorophenyl)ethanone $(1.0 \mathrm{mmol})$. The reaction mixture was stirred for $2 \mathrm{~h}$. Progress of the reaction was monitored by TLC. After completion, the mixture was poured into water and the precipitated solid was filtered, dried and recrystallized (EtOAc/hexane) to afford 2-(4-chlorophenyl)-2oxoethyl 3-bromobenzoate (1). The formation of keto ester (3) was indicated by the appearance of two typical stretching vibrations $v(\mathrm{C}=\mathrm{O})$ ester $(1724)$ and $v(\mathrm{C}=\mathrm{O})$ keto $(1698) \mathrm{cm}^{-1}$, respectively and the disappearance of characteristic IR stretching absorptions ascribable to the carboxylic acid group in the region of $3400-2400 \mathrm{~cm}^{-1}$. In the ${ }^{1} \mathrm{H}$ NMR spectrum, the signals for the aromatic protons appeared in their respective regions and the disappearance of a characteristic signal for the $\mathrm{COOH}$ proton confirmed the formation of the title compound (1). The ${ }^{13} \mathrm{C}$ NMR spectrum displayed two characteristic signals for the keto and ester carbonyl carbon atoms at 190.7 and 165.3 p.p.m., respectively. Yield: $88 \%$; m.p. 372-373 K; $R_{\mathrm{f}}: 0.72$ (10\% EtOAc/hexane); IR (ATR, $\mathrm{cm}^{-1}$ ): $3089\left(\mathrm{Csp}^{2}-\mathrm{H}\right), 2933,2856\left(\mathrm{Csp}^{3}-\mathrm{H}\right), 1724$ (C=O ester), 1698 $(\mathrm{C}=\mathrm{O}$ ketone $), 1585,1479(\mathrm{C}=\mathrm{C}$ Ar $), 1225(\mathrm{C}-\mathrm{O}) ;{ }^{1} \mathrm{H}$ NMR $\left(300 \mathrm{MHz}, \mathrm{CDCl}_{3}\right): \delta 8.06-8.03(\mathrm{~m}, 1 \mathrm{H}, \mathrm{Ar}-\mathrm{H}), 7.94-7.90(\mathrm{~m}$, 2H, Ar-H), 7.73-7.70 ( $m, 1 \mathrm{H}, \mathrm{Ar}-\mathrm{H}), 7.52-7.48$ ( $m, 2 \mathrm{H}, \mathrm{Ar}-\mathrm{H})$, 7.46-7.36 (m, 2H, Ar-H), $5.57\left(s, 2 \mathrm{H}, \mathrm{CH}_{2}\right) ;{ }^{13} \mathrm{C} \mathrm{NMR}$

Table 2

Experimental details.

Crystal data

Chemical formula

$M_{\mathrm{r}}$

Crystal system, space group

Temperature (K)

$a, b, c(\AA)$

$\alpha, \beta, \gamma\left({ }^{\circ}\right)$

$V\left(\AA^{3}\right)$

$Z$

Radiation type

$\mu\left(\mathrm{mm}^{-1}\right)$

Crystal size (mm)

Data collection

Diffractometer

Absorption correction

$T_{\min }, T_{\max }$

No. of measured, independent and observed $[I>2 \sigma(I)]$ reflections

$R_{\text {int }}$

$(\sin \theta / \lambda)_{\max }\left(\AA^{-1}\right)$

Refinement

$R\left[F^{2}>2 \sigma\left(F^{2}\right)\right], w R\left(F^{2}\right), S$

No. of reflections

No. of parameters

$\mathrm{H}$-atom treatment

$\Delta \rho_{\max }, \Delta \rho_{\min }\left(\mathrm{e} \AA^{-3}\right)$

Computer programs: CrysAlis PRO, (Agilent, 2011), SHELXS97 and SHELXL2014 (Sheldrick, 2008), Mercury (Macrae et al., 2008), enCIFer (Allen et al., 2004), PLATON (Spek, 2009) and publCIF (Westrip, 2010).

$\left(75 \mathrm{MHz}, \mathrm{CDCl}_{3}\right): \delta 190.7,165.3,140.6,134.5,133.1,132.4$, $132.0,131.0,129.3,129.3,127.3,122.1,66.5$.

\section{Refinement}

All $\mathrm{H}$ atoms were refined using a riding model, with $\mathrm{C}-\mathrm{H}=$ $0.95 \AA$ and $U_{\text {iso }}(\mathrm{H})=1.2 U_{\text {eq }}(\mathrm{C})$ for aromatic, and $\mathrm{C}-\mathrm{H}=$ $0.99 \AA$ and $U_{\text {iso }}(\mathrm{H})=1.2 U_{\text {eq }}(\mathrm{C})$ for the methylene $\mathrm{H}$ atoms.

\section{Acknowledgements}

We thank the Chemistry Department, University of Otago, for support of the work of JS.

\section{References}

Agilent (2011). CrysAlis PRO. Agilent Technologies, Yarnton, England.

Allen, F. H., Johnson, O., Shields, G. P., Smith, B. R. \& Towler, M. (2004). J. Appl. Cryst. 37, 335-338.

Chidan Kumar, C. S., Chia, T. S., Chandraju, S., Ooi, C. W., Quah, C. H. \& Fun, H.-K. (2014c). Z. Kristallogr. 229, 328-342.

Chidan Kumar, C. S., Yohannan Panicker, C., Fun, H.-K., Sheena Mary, Y., Harikumar, B., Chandraju, S., Quah, C. H. \& Ooi, C. W. (2014a). Spectrochim. Acta Part A, 126, 208-219.

Chidan Kumar, C. S., Yohannan Panicker, C., Fun, H.-K., Sheena Mary, Y., Harikumar, B., Chandraju, S., Quah, C. H. \& Ooi, C. W. (2014b). Spectrochim. Acta Part A, 128, 327-336.

Fun, H.-K., Arshad, S., Garudachari, B., Isloor, A. M. \& Satyanarayan, M. N. (2011a). Acta Cryst. E67, o1528. 
Fun, H.-K., Loh, W.-S., Garudachari, B., Isloor, A. M. \& Satyanarayan, M. N. (2011b). Acta Cryst. E67, o1597.

Groom, C. R. \& Allen, F. H. (2014). Angew. Chem. Int. Ed. Engl. 53, 662-671.

Kai, K., Yano, F., Suzuki, F., Kitagawa, H., Suzuki, M., Yokoyama, M. \& Watanabe, N. (2007). Tetrahedron, 63, 10630-10636.

Khan, I., Ibrar, A., Korzański, A. \& Kubicki, M. (2012). Acta Cryst. E68, o3465.

Kinoshita, M. \& Umezawa, S. (1960). Bull. Chem. Soc. Jpn, 33, 10751080.

Macrae, C. F., Bruno, I. J., Chisholm, J. A., Edgington, P. R., McCabe, P., Pidcock, E., Rodriguez-Monge, L., Taylor, R., van de Streek, J. \& Wood, P. A. (2008). J. Appl. Cryst. 41, 466-470.
Pal, S., Mareddy, J. \& Devi, N. S. (2008). J. Braz. Chem. Soc. 19, $1207-$ 1214.

Sheibani, H., Islami, M. R., Hassanpour, A. \& Hosseininasab, F. A. (2006b). ARKIVOC, 15, 68-75.

Sheibani, H., Islami, M. R., Hassanpour, A. \& Saidi, K. (2007). Phosphorus Sulfur Silicon Relat. Elem. 183, 13-20.

Sheibani, H., Mosslemin, M. H., Behzadi, S., Islami, M. R. \& Saidi, K. (2006a). Synthesis, 3, 435-439.

Sheldrick, G. M. (2008). Acta Cryst. A64, 112-122.

Spek, A. L. (2009). Acta Cryst. D65, 148-155.

Stanovnik, B. \& Svete, J. (2004). Chem. Rev. 104, 2433-2480.

Westrip, S. P. (2010). J. Appl. Cryst. 43, 920-925.

Zhang, L., Shen, Y., Zhu, H. J., Wang, F., Leng, Y. \& Liu, J. K. (2009). J. Antibiot. 62, 239-242. 


\section{supporting information}

Acta Cryst. (2014). E70, 301-304 [doi:10.1107/S1600536814021643]

\section{Crystal structure of 2-(4-chlorophenyl)-2-oxoethyl 3-bromobenzoate}

Imtiaz Khan, Aliya Ibrar, Shahid Hameed, Jonathan M. White and Jim Simpson

\section{Computing details}

Data collection: CrysAlis PRO, (Agilent, 2011); cell refinement: CrysAlis PRO, (Agilent, 2011); data reduction: CrysAlis PRO, (Agilent, 2011); program(s) used to solve structure: SHELXS97 (Sheldrick, 2008); program(s) used to refine structure: SHELXL2014 (Sheldrick, 2008); molecular graphics: Mercury (Macrae et al., 2008); software used to prepare material for publication: SHELXL2014 (Sheldrick, 2008), enCIFer (Allen et al., 2004), PLATON (Spek, 2009) and publCIF (Westrip, 2010).

\section{2-(4-Chlorophenyl)-2-oxoethyl 3-bromobenzoate}

Crystal data

$\mathrm{C}_{15} \mathrm{H}_{10} \mathrm{BrClO}_{3}$

$M_{r}=353.59$

Triclinic, $P \overline{1}$

$a=6.6797(3) \AA$

$b=10.0238(4) \AA$

$c=10.7851(5) \AA$

$\alpha=90.980(4)^{\circ}$

$\beta=107.573(4)^{\circ}$

$\gamma=92.138(3)^{\circ}$

$V=687.64(5) \AA^{3}$

\section{Data collection}

Agilent SuperNova (Dual, $\mathrm{Cu}$ at zero, Atlas

CCD)

diffractometer

Radiation source: SuperNova (Mo) X-ray

Source

Mirror monochromator

$\omega$ scans

Absorption correction: multi-scan

(CrysAlis PRO; Agilent, 2011)

\section{Refinement}

Refinement on $F^{2}$

Least-squares matrix: full

$R\left[F^{2}>2 \sigma\left(F^{2}\right)\right]=0.027$

$w R\left(F^{2}\right)=0.064$

$S=1.05$

3327 reflections

181 parameters

0 restraints
$Z=2$

$F(000)=352$

$D_{\mathrm{x}}=1.708 \mathrm{Mg} \mathrm{m}^{-3}$

Mo $K \alpha$ radiation, $\lambda=0.71073 \AA$

Cell parameters from 6648 reflections

$\theta=3.2-29.2^{\circ}$

$\mu=3.19 \mathrm{~mm}^{-1}$

$T=130 \mathrm{~K}$

Block, colourless

$0.50 \times 0.40 \times 0.20 \mathrm{~mm}$

$T_{\min }=0.504, T_{\max }=1.000$

10822 measured reflections

3327 independent reflections

3062 reflections with $I>2 \sigma(I)$

$R_{\text {int }}=0.033$

$\theta_{\max }=29.2^{\circ}, \theta_{\min }=3.2^{\circ}$

$h=-8 \rightarrow 8$

$k=-13 \rightarrow 13$

$l=-14 \rightarrow 14$

Hydrogen site location: inferred from neighbouring sites

$\mathrm{H}$-atom parameters constrained

$w=1 /\left[\sigma^{2}\left(F_{\mathrm{o}}^{2}\right)+(0.0307 P)^{2}+0.1071 P\right]$

where $P=\left(F_{\mathrm{o}}{ }^{2}+2 F_{\mathrm{c}}{ }^{2}\right) / 3$

$(\Delta / \sigma)_{\max }=0.001$

$\Delta \rho_{\max }=0.35 \mathrm{e} \AA^{-3}$

$\Delta \rho_{\min }=-0.60$ e $\AA^{-3}$ 
Special details

Experimental. Absorption correction: CrysAlisPro, Agilent (2011), Empirical absorption correction using spherical harmonics, implemented in SCALE3 ABSPACK scaling algorithm.

Geometry. All e.s.d.'s (except the e.s.d. in the dihedral angle between two 1.s. planes) are estimated using the full covariance matrix. The cell e.s.d.'s are taken into account individually in the estimation of e.s.d.'s in distances, angles and torsion angles; correlations between e.s.d.'s in cell parameters are only used when they are defined by crystal symmetry. An approximate (isotropic) treatment of cell e.s.d.'s is used for estimating e.s.d.'s involving 1.s. planes.

Fractional atomic coordinates and isotropic or equivalent isotropic displacement parameters $\left(\AA^{2}\right)$

\begin{tabular}{|c|c|c|c|c|}
\hline & $x$ & $y$ & $z$ & $U_{\text {iso }} * / U_{\text {eq }}$ \\
\hline $\mathrm{C} 1$ & $0.1452(3)$ & $0.76183(16)$ & $0.09834(16)$ & $0.0153(3)$ \\
\hline $\mathrm{C} 2$ & $-0.0463(3)$ & $0.73719(17)$ & $0.00240(16)$ & $0.0165(3)$ \\
\hline $\mathrm{H} 2$ & -0.0671 & 0.6625 & -0.0562 & $0.020^{*}$ \\
\hline $\mathrm{C} 3$ & $-0.2052(3)$ & $0.82374(17)$ & $-0.00564(16)$ & 0.0164 \\
\hline $\mathrm{C} 4$ & $-0.1816(3)$ & 0.93075 (18) & $0.08011(17)$ & $0.0200(4)$ \\
\hline $\mathrm{H} 4$ & -0.2944 & 0.9879 & 0.0739 & $0.024 *$ \\
\hline $\mathrm{C} 5$ & $0.0097(3)$ & $0.95371(18)$ & $0.17577(17)$ & $0.0213(4)$ \\
\hline H5 & 0.0280 & 1.0271 & 0.2356 & $0.026^{*}$ \\
\hline C6 & $0.1742(3)$ & $0.87044(17)$ & $0.18485(17)$ & $0.0189(3)$ \\
\hline H6 & 0.3057 & 0.8874 & 0.2496 & $0.023 *$ \\
\hline $\mathrm{C} 7$ & $0.3120(3)$ & $0.66531(17)$ & $0.10555(16)$ & $0.0169(3)$ \\
\hline $\mathrm{C} 8$ & $0.6639(3)$ & $0.62000(17)$ & $0.20718(17)$ & $0.0182(3)$ \\
\hline $\mathrm{H} 8 \mathrm{~A}$ & 0.8031 & 0.6678 & 0.2388 & $0.022 *$ \\
\hline H8B & 0.6573 & 0.5687 & 0.1266 & $0.022 *$ \\
\hline $\mathrm{C} 9$ & $0.6368(3)$ & $0.52539(17)$ & $0.30950(16)$ & $0.0176(3)$ \\
\hline $\mathrm{C} 10$ & $0.8001(3)$ & $0.42573(17)$ & $0.35889(16)$ & $0.0166(3)$ \\
\hline $\mathrm{C} 11$ & $0.9739(3)$ & $0.41710(18)$ & $0.31370(17)$ & $0.0201(4)$ \\
\hline H11 & 0.9919 & 0.4770 & 0.2502 & $0.024 *$ \\
\hline $\mathrm{C} 12$ & $1.1213(3)$ & $0.32135(19)$ & $0.36109(18)$ & $0.0231(4)$ \\
\hline $\mathrm{H} 12$ & 1.2400 & 0.3152 & 0.3305 & $0.028^{*}$ \\
\hline $\mathrm{C} 13$ & $1.0919(3)$ & $0.23586(18)$ & $0.45295(18)$ & $0.0229(4)$ \\
\hline $\mathrm{C} 14$ & $0.9204(3)$ & $0.24247(19)$ & $0.49972(18)$ & $0.0242(4)$ \\
\hline $\mathrm{H} 14$ & 0.9029 & 0.1821 & 0.5630 & $0.029 *$ \\
\hline $\mathrm{C} 15$ & $0.7757(3)$ & $0.33824(19)$ & $0.45280(17)$ & $0.0215(4)$ \\
\hline H15 & 0.6584 & 0.3446 & 0.4848 & $0.026^{*}$ \\
\hline $\mathrm{O} 1$ & $0.28187(18)$ & $0.55756(12)$ & $0.05094(12)$ & $0.0222(3)$ \\
\hline $\mathrm{O} 2$ & 0.50195 (18) & 0.71409 (12) & $0.17991(12)$ & $0.0186(3)$ \\
\hline $\mathrm{O} 3$ & $0.48450(19)$ & 0.53074 (14) & $0.34811(13)$ & $0.0257(3)$ \\
\hline $\mathrm{Cl1}$ & $1.27582(8)$ & $0.11559(5)$ & $0.51284(5)$ & $0.03372(13)$ \\
\hline Br1 & $-0.46435(2)$ & $0.79269(2)$ & $-0.13927(2)$ & $0.02456(7)$ \\
\hline
\end{tabular}

Atomic displacement parameters $\left(\AA^{2}\right)$

\begin{tabular}{lllllll}
\hline & $U^{11}$ & $U^{22}$ & $U^{33}$ & $U^{12}$ & $U^{13}$ & $U^{23}$ \\
\hline $\mathrm{C} 1$ & $0.0181(8)$ & $0.0132(8)$ & $0.0156(8)$ & $0.0008(6)$ & $0.0067(7)$ & $0.0013(6)$ \\
$\mathrm{C} 2$ & $0.0189(8)$ & $0.0136(8)$ & $0.0178(8)$ & $-0.0008(6)$ & $0.0069(7)$ & $-0.0010(6)$ \\
$\mathrm{C} 3$ & $0.0165(8)$ & $0.0160(9)$ & $0.0160(8)$ & $-0.0002(6)$ & $0.0039(7)$ & $0.0007(6)$
\end{tabular}


supporting information

$\begin{array}{lllllll}\text { C4 } & 0.0244(9) & 0.0160(9) & 0.0213(9) & 0.0033(7) & 0.0091(7) & -0.0003(7) \\ \text { C5 } & 0.0288(9) & 0.0157(9) & 0.0196(9) & 0.0016(7) & 0.0078(8) & -0.0043(7) \\ \text { C6 } & 0.0219(8) & 0.0167(9) & 0.0164(8) & -0.0014(7) & 0.0035(7) & -0.0012(6) \\ \text { C7 } & 0.0166(8) & 0.0178(9) & 0.0159(8) & -0.0010(6) & 0.0043(7) & 0.0009(6) \\ \text { C8 } & 0.0159(8) & 0.0179(9) & 0.0206(9) & 0.0023(6) & 0.0049(7) & -0.0008(7) \\ \text { C9 } & 0.0181(8) & 0.0192(9) & 0.0142(8) & -0.0007(6) & 0.0035(7) & -0.0043(6) \\ \text { C10 } & 0.0179(8) & 0.0154(9) & 0.0144(8) & -0.0023(6) & 0.0021(7) & -0.0035(6) \\ \text { C11 } & 0.0217(8) & 0.0187(9) & 0.0204(9) & 0.0004(7) & 0.0071(7) & -0.0001(7) \\ \text { C12 } & 0.0187(8) & 0.0241(10) & 0.0256(10) & 0.0009(7) & 0.0055(8) & -0.0029(7) \\ \text { C13 } & 0.0258(9) & 0.0169(9) & 0.0188(9) & 0.0036(7) & -0.0041(7) & -0.0050(7) \\ \text { C14 } & 0.0314(10) & 0.0206(10) & 0.0181(9) & -0.0022(7) & 0.0037(8) & 0.0015(7) \\ \text { C15 } & 0.0227(9) & 0.0238(10) & 0.0174(9) & -0.0021(7) & 0.0058(7) & -0.0024(7) \\ \text { O1 } & 0.0204(6) & 0.0167(7) & 0.0272(7) & 0.0018(5) & 0.0043(5) & -0.0065(5) \\ \text { O2 } & 0.0154(6) & 0.0162(6) & 0.0218(6) & 0.0014(5) & 0.0019(5) & -0.0017(5) \\ \text { O3 } & 0.0224(6) & 0.0325(8) & 0.0262(7) & 0.0046(5) & 0.0127(6) & 0.0037(6) \\ \text { C11 } & 0.0352(3) & 0.0251(3) & 0.0316(3) & 0.0110(2) & -0.0049(2) & -0.0012(2) \\ \text { Br1 } & 0.01750(10) & 0.02591(12) & 0.02626(12) & 0.00457(7) & 0.00057(8) & -0.00652(8) \\ & & & & & & \end{array}$

Geometric parameters $\left(\AA,{ }^{\circ}\right)$

\begin{tabular}{llll}
\hline $\mathrm{C} 1-\mathrm{C} 6$ & $1.391(2)$ & $\mathrm{C} 8-\mathrm{H} 8 \mathrm{~A}$ & 0.9900 \\
$\mathrm{C} 1-\mathrm{C} 2$ & $1.391(2)$ & $\mathrm{C} 8-\mathrm{H} 8 \mathrm{~B}$ & 0.9900 \\
$\mathrm{C} 1-\mathrm{C} 7$ & $1.488(2)$ & $\mathrm{C} 9-\mathrm{O} 3$ & $1.212(2)$ \\
$\mathrm{C} 2-\mathrm{C} 3$ & $1.380(2)$ & $\mathrm{C} 9-\mathrm{C} 10$ & $1.490(2)$ \\
$\mathrm{C} 2-\mathrm{H} 2$ & 0.9500 & $\mathrm{C} 10-\mathrm{C} 11$ & $1.393(2)$ \\
$\mathrm{C} 3-\mathrm{C} 4$ & $1.377(2)$ & $\mathrm{C} 10-\mathrm{C} 15$ & $1.393(2)$ \\
$\mathrm{C} 3-\mathrm{B} 1$ & $1.8995(16)$ & $\mathrm{C} 11-\mathrm{C} 12$ & $1.391(2)$ \\
$\mathrm{C} 4-\mathrm{C} 5$ & $1.387(3)$ & $\mathrm{C} 11-\mathrm{H} 11$ & 0.9500 \\
$\mathrm{C} 4-\mathrm{H} 4$ & 0.9500 & $\mathrm{C} 12-\mathrm{C} 13$ & $1.374(3)$ \\
$\mathrm{C} 5-\mathrm{C} 6$ & $1.386(2)$ & $\mathrm{C} 12-\mathrm{H} 12$ & 0.9500 \\
$\mathrm{C} 5-\mathrm{H} 5$ & 0.9500 & $\mathrm{C} 13-\mathrm{C} 14$ & $1.387(3)$ \\
$\mathrm{C} 6-\mathrm{H} 6$ & 0.9500 & $\mathrm{C} 13-\mathrm{C} 11$ & $1.7429(18)$ \\
$\mathrm{C} 7-\mathrm{O} 1$ & $1.202(2)$ & $\mathrm{C} 14-\mathrm{C} 15$ & $1.379(3)$ \\
$\mathrm{C} 7-\mathrm{O} 2$ & $1.348(2)$ & $\mathrm{C} 14-\mathrm{H} 14$ & 0.9500 \\
$\mathrm{C} 8-\mathrm{O} 2$ & $1.4283(19)$ & $\mathrm{C} 15-\mathrm{H} 15$ & 0.9500 \\
$\mathrm{C} 8-\mathrm{C} 9$ & $1.515(2)$ & & 109.7 \\
$\mathrm{C} 6-\mathrm{C} 1-\mathrm{C} 2$ & & $\mathrm{C} 9-\mathrm{C} 8-\mathrm{H} 8 \mathrm{~B}$ & 108.2 \\
$\mathrm{C} 6-\mathrm{C} 1-\mathrm{C} 7$ & $120.59(15)$ & $\mathrm{H} 8 \mathrm{~A}-\mathrm{C} 8-\mathrm{H} 8 \mathrm{~B}$ & $121.67(15)$ \\
$\mathrm{C} 2-\mathrm{C} 1-\mathrm{C} 7$ & $122.35(15)$ & $\mathrm{O} 3-\mathrm{C} 9-\mathrm{C} 10$ & $120.25(15)$ \\
$\mathrm{C} 3-\mathrm{C} 2-\mathrm{C} 1$ & $117.04(15)$ & $\mathrm{O} 3-\mathrm{C} 9-\mathrm{C} 8$ & $118.08(14)$ \\
$\mathrm{C} 3-\mathrm{C} 2-\mathrm{H} 2$ & $118.46(16)$ & $\mathrm{C} 10-\mathrm{C} 9-\mathrm{C} 8$ & $119.46(16)$ \\
$\mathrm{C} 1-\mathrm{C} 2-\mathrm{H} 2$ & 120.8 & $\mathrm{C} 11-\mathrm{C} 10-\mathrm{C} 15$ & $122.21(15)$ \\
$\mathrm{C} 4-\mathrm{C} 3-\mathrm{C} 2$ & 120.8 & $\mathrm{C} 11-\mathrm{C} 10-\mathrm{C} 9$ & $118.33(15)$ \\
$\mathrm{C} 4-\mathrm{C} 3-\mathrm{Br} 1$ & $122.08(16)$ & $\mathrm{C} 12-\mathrm{C} 10-\mathrm{C} 11-\mathrm{C} 10$ & $120.38(16)$ \\
$\mathrm{C} 2-\mathrm{C} 3-\mathrm{Br} 1$ & $119.44(13)$ & $\mathrm{C} 10-\mathrm{C} 11-\mathrm{H} 11$ & 119.8 \\
$\mathrm{C} 3-\mathrm{C} 4-\mathrm{C} 5$ & $118.49(13)$ & 119.8 \\
$\mathrm{C} 3-\mathrm{C} 4-\mathrm{H} 4$ & $120.85(16)$ & &
\end{tabular}




\begin{tabular}{|c|c|c|c|}
\hline $\mathrm{C} 5-\mathrm{C} 4-\mathrm{H} 4$ & 120.6 & $\mathrm{C} 13-\mathrm{C} 12-\mathrm{C} 11$ & $118.76(17)$ \\
\hline $\mathrm{C} 6-\mathrm{C} 5-\mathrm{C} 4$ & $120.58(17)$ & $\mathrm{C} 13-\mathrm{C} 12-\mathrm{H} 12$ & 120.6 \\
\hline $\mathrm{C} 6-\mathrm{C} 5-\mathrm{H} 5$ & 119.7 & $\mathrm{C} 11-\mathrm{C} 12-\mathrm{H} 12$ & 120.6 \\
\hline $\mathrm{C} 4-\mathrm{C} 5-\mathrm{H} 5$ & 119.7 & $\mathrm{C} 12-\mathrm{C} 13-\mathrm{C} 14$ & $122.01(17)$ \\
\hline $\mathrm{C} 5-\mathrm{C} 6-\mathrm{C} 1$ & $119.41(16)$ & $\mathrm{C} 12-\mathrm{C} 13-\mathrm{Cl1}$ & $119.05(15)$ \\
\hline $\mathrm{C} 5-\mathrm{C} 6-\mathrm{H} 6$ & 120.3 & $\mathrm{C} 14-\mathrm{C} 13-\mathrm{Cl} 1$ & $118.94(14)$ \\
\hline $\mathrm{C} 1-\mathrm{C} 6-\mathrm{H} 6$ & 120.3 & $\mathrm{C} 15-\mathrm{C} 14-\mathrm{C} 13$ & $118.85(17)$ \\
\hline $\mathrm{O} 1-\mathrm{C} 7-\mathrm{O} 2$ & $124.03(15)$ & $\mathrm{C} 15-\mathrm{C} 14-\mathrm{H} 14$ & 120.6 \\
\hline $\mathrm{O} 1-\mathrm{C} 7-\mathrm{C} 1$ & $124.27(15)$ & $\mathrm{C} 13-\mathrm{C} 14-\mathrm{H} 14$ & 120.6 \\
\hline $\mathrm{O} 2-\mathrm{C} 7-\mathrm{C} 1$ & $111.69(15)$ & $\mathrm{C} 14-\mathrm{C} 15-\mathrm{C} 10$ & $120.54(17)$ \\
\hline $\mathrm{O} 2-\mathrm{C} 8-\mathrm{C} 9$ & $109.80(13)$ & $\mathrm{C} 14-\mathrm{C} 15-\mathrm{H} 15$ & 119.7 \\
\hline $\mathrm{O} 2-\mathrm{C} 8-\mathrm{H} 8 \mathrm{~A}$ & 109.7 & $\mathrm{C} 10-\mathrm{C} 15-\mathrm{H} 15$ & 119.7 \\
\hline $\mathrm{C} 9-\mathrm{C} 8-\mathrm{H} 8 \mathrm{~A}$ & 109.7 & $\mathrm{C} 7-\mathrm{O} 2-\mathrm{C} 8$ & $114.81(13)$ \\
\hline $\mathrm{O} 2-\mathrm{C} 8-\mathrm{H} 8 \mathrm{~B}$ & 109.7 & & \\
\hline $\mathrm{C} 6-\mathrm{C} 1-\mathrm{C} 2-\mathrm{C} 3$ & $0.7(2)$ & $\mathrm{C} 8-\mathrm{C} 9-\mathrm{C} 10-\mathrm{C} 11$ & $-0.3(2)$ \\
\hline $\mathrm{C} 7-\mathrm{C} 1-\mathrm{C} 2-\mathrm{C} 3$ & $179.07(14)$ & $\mathrm{O} 3-\mathrm{C} 9-\mathrm{C} 10-\mathrm{C} 15$ & $-0.7(3)$ \\
\hline $\mathrm{C} 1-\mathrm{C} 2-\mathrm{C} 3-\mathrm{C} 4$ & $-1.8(2)$ & $\mathrm{C} 8-\mathrm{C} 9-\mathrm{C} 10-\mathrm{C} 15$ & $-179.85(16)$ \\
\hline $\mathrm{C} 1-\mathrm{C} 2-\mathrm{C} 3-\mathrm{Br} 1$ & $178.47(11)$ & $\mathrm{C} 15-\mathrm{C} 10-\mathrm{C} 11-\mathrm{C} 12$ & $0.5(3)$ \\
\hline $\mathrm{C} 2-\mathrm{C} 3-\mathrm{C} 4-\mathrm{C} 5$ & $1.4(3)$ & $\mathrm{C} 9-\mathrm{C} 10-\mathrm{C} 11-\mathrm{C} 12$ & $-179.10(16)$ \\
\hline $\mathrm{Br} 1-\mathrm{C} 3-\mathrm{C} 4-\mathrm{C} 5$ & $-178.85(12)$ & $\mathrm{C} 10-\mathrm{C} 11-\mathrm{C} 12-\mathrm{C} 13$ & $0.0(3)$ \\
\hline $\mathrm{C} 3-\mathrm{C} 4-\mathrm{C} 5-\mathrm{C} 6$ & $0.1(3)$ & $\mathrm{C} 11-\mathrm{C} 12-\mathrm{C} 13-\mathrm{C} 14$ & $-0.1(3)$ \\
\hline $\mathrm{C} 4-\mathrm{C} 5-\mathrm{C} 6-\mathrm{C} 1$ & $-1.1(3)$ & $\mathrm{C} 11-\mathrm{C} 12-\mathrm{C} 13-\mathrm{C} 11$ & $180.00(14)$ \\
\hline $\mathrm{C} 2-\mathrm{C} 1-\mathrm{C} 6-\mathrm{C} 5$ & $0.7(2)$ & $\mathrm{C} 12-\mathrm{C} 13-\mathrm{C} 14-\mathrm{C} 15$ & $-0.3(3)$ \\
\hline $\mathrm{C} 7-\mathrm{C} 1-\mathrm{C} 6-\mathrm{C} 5$ & $-177.59(15)$ & $\mathrm{C} 11-\mathrm{C} 13-\mathrm{C} 14-\mathrm{C} 15$ & $179.61(14)$ \\
\hline $\mathrm{C} 6-\mathrm{C} 1-\mathrm{C} 7-\mathrm{O} 1$ & $164.73(17)$ & $\mathrm{C} 13-\mathrm{C} 14-\mathrm{C} 15-\mathrm{C} 10$ & $0.8(3)$ \\
\hline $\mathrm{C} 2-\mathrm{C} 1-\mathrm{C} 7-\mathrm{O} 1$ & $-13.6(2)$ & $\mathrm{C} 11-\mathrm{C} 10-\mathrm{C} 15-\mathrm{C} 14$ & $-0.9(3)$ \\
\hline $\mathrm{C} 6-\mathrm{C} 1-\mathrm{C} 7-\mathrm{O} 2$ & $-15.9(2)$ & $\mathrm{C} 9-\mathrm{C} 10-\mathrm{C} 15-\mathrm{C} 14$ & $178.71(16)$ \\
\hline $\mathrm{C} 2-\mathrm{C} 1-\mathrm{C} 7-\mathrm{O} 2$ & $165.76(13)$ & $\mathrm{O} 1-\mathrm{C} 7-\mathrm{O} 2-\mathrm{C} 8$ & $-9.5(2)$ \\
\hline $\mathrm{O} 2-\mathrm{C} 8-\mathrm{C} 9-\mathrm{O} 3$ & $5.4(2)$ & $\mathrm{C} 1-\mathrm{C} 7-\mathrm{O} 2-\mathrm{C} 8$ & $171.15(13)$ \\
\hline $\mathrm{O} 2-\mathrm{C} 8-\mathrm{C} 9-\mathrm{C} 10$ & $-175.45(14)$ & $\mathrm{C} 9-\mathrm{C} 8-\mathrm{O} 2-\mathrm{C} 7$ & $-75.86(17)$ \\
\hline $\mathrm{O} 3-\mathrm{C} 9-\mathrm{C} 10-\mathrm{C} 11$ & $178.89(17)$ & & \\
\hline
\end{tabular}

Hydrogen-bond geometry $\left(\AA,{ }^{\circ}\right)$

\begin{tabular}{lllll}
\hline$D-\mathrm{H} \cdots A$ & $D-\mathrm{H}$ & $\mathrm{H} \cdots A$ & $D \cdots A$ & $D-\mathrm{H} \cdots A$ \\
\hline $\mathrm{C} 4-\mathrm{H} 4 \cdots \mathrm{Br}^{\mathrm{i}}$ & 0.95 & 2.97 & $3.8762(18)$ & 160 \\
$\mathrm{C} 8-\mathrm{H} 8 B \cdots{ }^{\mathrm{ii}}$ & 0.99 & 2.42 & $3.396(2)$ & 168 \\
$\mathrm{C} 15-\mathrm{H} 15 \cdots{ }^{\mathrm{ii}}{ }^{\mathrm{ii}}$ & 0.95 & 2.60 & $3.418(2)$ & 144 \\
\hline
\end{tabular}

Symmetry codes: (i) $-x-1,-y+2,-z$; (ii) $-x+1,-y+1,-z$; (iii) $-x+1,-y+1,-z+1$. 